\title{
On the method of changing the temperature of liquids and gases in a transportation system by local heating or cooling
}

\author{
Evgeny L. Pankratov ${ }^{1,2}$ \\ ${ }^{1}$ Nizhny Novgorod State University, 23 Gagarin avenue, Nizhny Novgorod, 603950, Russia. \\ ${ }^{2}$ Nizhny Novgorod State Technical University, 24 Minin Street, Nizhny Novgorod, 603950, Russia.
}

\begin{abstract}
In this paper, we consider an approach to changing the temperature of liquids and gases in a pipeline by local heating and cooling. The concept is based on the introduction of changes in the temperature series of the pipeline sections due to the external modification these sections' temperature. This situation could be used as a heat sink or to increase temperature. A developed heat transfer model in a pipeline also accounts for convection due to liquid/gas transport. An analytical approach for the analysis of liquid/gas transport that takes into account the transport of heat due to convection was also introduced. The approach allows the spatial and temporal variation of parameters of transport to be taken into account as well as considerations of the nonlinearity of the investigated processes.
\end{abstract}

Keywords: liquids and gases transport; heat transfer; local heating or cooling; analytical modelling.

\section{Introduction}

In several technical applications, it is necessary to make a heat sink and, if necessary, stabilize the temperature of the liquid or gaseous heat carrier. In the framework method, for the transport of heat carrier, a cylindrical transportation system is considered (a frequent example of such a system is a pipeline) with a circular section (see Fig. 1). This pipeline has a metal section with a known dimension and a porous metal inside. This section has a stable temperature (for example, the section is located in a stream of water at the required temperature) to correct gas or liquid temperature to the required value. The main aim of the paper is to estimate spatio-temporal distribution of temperature in the considered pipeline under the influence of the introduced metal sections with a sta- ble temperature. The accompanying aim of the present paper is the creation of a model of the considered mass and heat transport, as well as an introduction of an analytical approach for the prognosis of mass and heat transport.

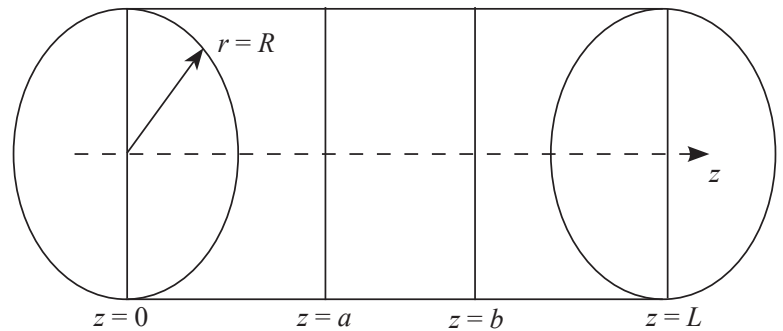

Fig. 1. Structure of the considered pipe is $L$ (axial coordinate $z \in[0, L]$ ) with radius $R$. This tube has a section $z \in[a, b]$

*Author' e-mail: elp2004@mail.ru

ORCID ID: 0000-0001-6174-2612

(c) 2020 Author. This is an open access publication, which can be used, distributed and reproduced in any medium according to the Creative Commons CC-BY 4.0 License requiring that the original work has been properly cited. 


\section{Method of solution}

To solve the defined aims, the spatio-temporal distribution of the considered temperature was calculated. The required distribution of temperature was calculated by the solution of the second Fourier law (Carslaw \& Jaeger, 1964; Fedorenko et al., 2020; Kalaev, 2020; Liu et al., 2020):

$$
\begin{gathered}
c \frac{\partial T(r, \varphi, z, t)}{\partial t}=\operatorname{div}\{\lambda \cdot \operatorname{grad}[T(r, \varphi, z, t)]- \\
\vec{v}(r, \varphi, z, t) \cdot c \cdot T(r, \varphi, z, t) \cdot C\}+p(r, z, t)
\end{gathered}
$$

Here $r, \varphi, z$ and $t$ are the current cylindrical coordinates and time $(r \in[0, R], \varphi \in[0,2 \pi], z \in[0, L])$; $T(r, \varphi, z, t)$ is the spatio-temporal distribution of temperature; $c$ is the specific heat capacity of the considered system; $C$ is the concentration of the transported substance in the pipeline; $\lambda$ is the coefficient of thermal conductivity; $p(r, z, t)$ is the density of power, which is stand out released in the considered system; $\vec{v}$ is the flow velocity of heat carrier, which is described by the Navier-Stokes equation:

$$
\frac{\partial \vec{v}}{\partial t}+(\vec{v} \cdot \nabla) \vec{v}=-\nabla\left(\frac{P}{C}\right)+v \Delta \vec{v}
$$

where $P$ is the pressure in the pipeline; $v$ is the kinematics viscosity. Boundary and initial conditions for the considered equations can be expressed as:

$$
\begin{gathered}
-\left.\lambda \frac{\partial T(r, \varphi, z<a, t)}{\partial r}\right|_{r=R}=0 \\
\left.\frac{\partial T(r, \varphi, z, t)}{\partial \varphi}\right|_{\varphi=0}=\left.\frac{\partial T(r, \varphi, z, t)}{\partial \varphi}\right|_{\varphi=2 \pi} \\
T(r, 0, z, t)=T(r, 2 \pi, z, t) ; T(0, \varphi, z, t) \neq \infty \\
T(r, \varphi, z, 0)=T_{0} ; T(r, \varphi, 0, t)=T(r, \varphi, L, t)=T_{b 1} \quad(3) \\
T(r, \varphi, z \in[a, b], t)=T_{b 2} \\
\left.\frac{\partial v_{\varphi}(r, \varphi, z, t)}{\partial \varphi}\right|_{\varphi=0}=\left.\frac{\partial v_{\varphi}(r, \varphi, z, t)}{\partial \varphi}\right|_{\varphi=2 \pi} \\
-\left.\lambda \frac{\partial T(r, \varphi, z>b, t)}{\partial r}\right|_{r=R}=\left.0 \frac{\partial v_{r}(r, \varphi, z, t)}{\partial r}\right|_{r=R}=0 \\
v_{\varphi}(r, 0, z, t)=v_{\varphi}(r, 2 \pi, z, t), v_{r}(0, \varphi, z, t) \neq \infty \\
v_{z}(r, \varphi, 0, t)=v_{z}(r, \varphi, L, t)=V_{0} \\
v_{r}(r, \varphi, z, t)=v_{\varphi}(r, \varphi, z, t)=v_{z}(r, \varphi, z, t)=V_{0}
\end{gathered}
$$

Due to the symmetry of the investigated system, to describe the velocity, one can only consider the axial velocity component, i.e. $v_{z}$. To solve equation $(4 \mathrm{c})$, the method of averaging functional corrections was used (Sokolov, 1955; Pankratov, 2012). It should be noted that the method provides the opportunity to obtain solutions to the above equations analytically in the more common case in comparison with recent approaches: Fourier approach, integral transformation approach (Carslaw \& Jaeger, 1964; Fedorenko et al., 2020). Numerical approaches for the solution of equations have less visibility in comparison with analytical ones (Kalaev, 2020; Liu et al., 2020). The method of averaging functional corrections provides the opportunity to con- 
sider the spatial and temporal variation of the parameters of transport in more common cases than by using Fourier and integral transformation approaches. At the same time, it is possible to take into account the nonlinearity of the considered processes. The framework method of averaging functional corrections to determine the first-order approximation of the projection of the velocity of the heat carrier flow, we replace it on not yet known average value (i.e. $v_{z} \rightarrow \alpha_{1 z}$ ) in the righthand side of equation (4c). After this substitution, the equations for the first-order approximations of the required component were obtained in the following form:

$$
\frac{\partial v_{1 z}}{\partial t}=-\frac{\partial}{\partial z}\left(\frac{P}{C}\right)
$$

The solution to this equation could be written as:

$$
v_{1 z}=-\frac{\partial}{\partial z} \int_{0}^{t} \frac{P}{C} d \tau
$$

The second-order approximation of the considered projection could be obtained by replacing the function on the right-hand side of equation (4c) with the following sum $v_{z} \rightarrow_{2 z}+v_{1 z}$. The equation for this projection is:

$v_{2 z}=v \int_{0}^{t} \frac{\partial^{2} v_{1 z}}{\partial z^{2}} d \tau-\int_{0}^{t}\left(\alpha_{2 z}+v_{1 z}\right) \frac{\partial v_{1 z}}{\partial z} d \tau$

The integration of this equation leads to the following result:

$$
v_{2 z}=v \int_{0}^{t} \frac{\partial^{2} v_{1 z}}{\partial z^{2}} d \tau-\int_{0}^{t}\left(\alpha_{2 z}+v_{1 z}\right) \frac{\partial v_{1 z}}{\partial z} d \tau
$$

The average value $\alpha_{2 z}$ was determined by the following standard relation:

$$
\alpha_{2 z}=\frac{1}{\Theta L} \int_{0}^{\Theta} \int_{0}^{L}\left(v_{2 z}-v_{1 z}\right) d z d t
$$

Here $\Theta$ is the continuance of observation on heat transport. Substitution of the first- and the second-order approximations of the considered velocity projection into relation (8) leads to the calculation of the required average value $\alpha_{2 z}$ and obtaining the following relation:

$$
\alpha_{2 z}=v \int_{0}^{\Theta}(\Theta-t) \int_{0}^{L} \frac{\partial^{2} v_{1 z}}{\partial z^{2}} d z d t
$$

In this section, the projection of the heat carrier flow velocity $v_{z}$ was calculated using the second-order approximation by the method of averaging functional corrections. Usually, the second-order approximation is a good enough approximation to make a qualitative analysis of the obtained solution and to obtain some quantitative estimates. Next, an equation (1) in a cylindrical coordinate system can be formulated:

$$
\begin{gathered}
c \frac{\partial T(r, z, t)}{\partial t}=\frac{1}{r} \frac{\partial}{\partial r}\left[r \lambda \frac{\partial T(r, z, t)}{\partial r}\right]+ \\
\frac{\partial}{\partial z}\left[\lambda \frac{\partial T(r, z, t)}{\partial z}\right]+p(r, z, t)+ \\
c \cdot C \frac{\partial v_{z}(z, t) \cdot T(r, z, t)}{\partial r}- \\
c \cdot C \frac{\partial v_{z}(z, t) \cdot T(r, z, t)}{\partial z}
\end{gathered}
$$

To determine the spatio-temporal distribution of temperature, again the same method of averaging functional corrections was used. To calculate the first-order approximation of the considered function, it is replaced by a not yet known average value $\alpha_{1 T}$ on the right side of equation (10). Using the above algorithm gives a possibility to obtain a relation for the first-order approximation of temperature in the following form:

$$
\begin{aligned}
T_{1}(r, z, t)= & T_{0}-\alpha_{1 T} C \int_{0}^{t} \frac{\partial v_{z}(z, \tau)}{\partial z} d \tau+ \\
& \frac{1}{c} \int_{0}^{t} p(r, z, \tau) d \tau
\end{aligned}
$$

Now it is possible to determine the not yet known average value $\alpha_{1 T}$ by using the following standard relation:

$$
\alpha_{1 T}=\frac{2}{\Theta R^{2} L} \int_{0}^{\Theta} \int_{0}^{R} r \int_{0}^{L} T_{1}(r, z, t) d z d r d t
$$

Substitution of the first-order approximation of temperature into relation (12) leads to the following result:

$$
\alpha_{1 T}=T_{0}+\frac{2}{c \Theta R^{2} L} \int_{0}^{\Theta}(\Theta-t) \int_{0}^{R} r \int_{0}^{L} p(r, z, t) d z d r d t
$$

We determine the second-order approximation framework by a standard procedure of the method of 
averaging of function corrections (Sokolov, 1955; Pankratov, 2012), i.e. by replacement of the required function in the right side of equation (12) by the following sum: $T \rightarrow \alpha_{2 T}+T_{1}$. In this case, the second-order approximation of the required function could be written as:

$$
\begin{gathered}
T_{2}(r, z, t)=\frac{1}{c r} \frac{\partial}{\partial r}\left[r \lambda \frac{\partial}{\partial r} \int_{0}^{t} T_{1}(r, z, \tau) d \tau\right]+ \\
\frac{1}{c} \frac{\partial}{\partial z}\left[\lambda \frac{\partial}{\partial z} \int_{0}^{t} T_{1}(r, z, \tau) d \tau\right]+ \\
\frac{1}{c} \int_{0}^{t} p(r, z, \tau) d \tau- \\
C \frac{\partial}{\partial r} \int_{0}^{t} v_{z}(z, \tau) \cdot T_{1}(r, z, \tau) d \tau- \\
C \frac{\partial}{\partial z} \int_{0}^{t} v_{z}(z, \tau) \cdot\left[\alpha_{2 T}+T_{1}(r, z, \tau)\right] d \tau
\end{gathered}
$$

An average value of the second-order approximation of temperature $\alpha_{2 T}$ can be calculated by the following standard relation:

$$
\alpha_{2 T}=\frac{2}{\Theta R^{2} L} \int_{0}^{\Theta} \int_{0}^{R} r \int_{0}^{L}\left(T_{2}-T_{1}\right) d z d r d t
$$

Substitution of the first- and the second-order approximation of temperature into relation (14) gives a relation for the required average value in the following form:

$$
\alpha_{2 T}=\frac{2}{c \Theta R^{2} L} \int_{0}^{\Theta}(\Theta-t) \int_{0}^{R} r \int_{0}^{L} p(r, z, t) d z d r d t
$$

Analysis of spatio-temporal distribution of temperature was done analytically by using the second-order approximation framework from the method of averaging functional corrections. The approximation is usually good enough to obtain a qualitative analysis and obtain some quantitative results. The results of analytical calculations were verified by comparing them with the results of numerical simulation.

\section{Discussion}

In this section, the temperature field in the system heat carrier - transportation system was analyzed. Figures 2-5 show the distributions of temperature in the considered system. Figures 2 and 3 show that changing the external temperature of the considered pipeline leads to chang- ing the internal temperature framework of the pipeline with a changing radius, which shows the adequacy of the model. Figures 4 and 5 show that changing of the temperature of an external porous section in considered pipeline leads to changing of internal temperature framework in the pipeline with changing of axial coordinate.

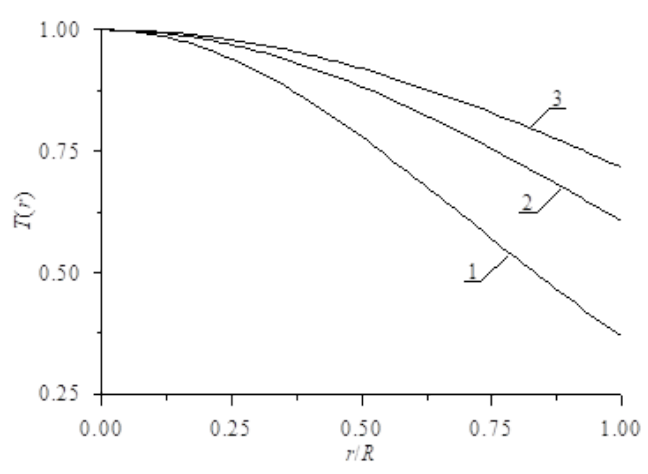

Fig. 2. The dependence of the temperature of the heat carrier on the radius at various values of the temperature of the wall of the transport system. The increasing number of the curves corresponds to the increase of the wall temperature, provided that it is less than the heat carrier temperature

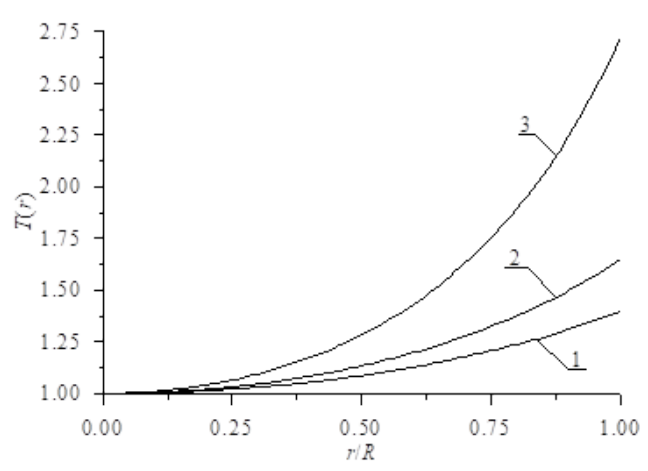

Fig. 3. The dependence of the temperature of the heat carrier on the radius at various values of the temperature of the wall of the transport system. The increasing number of the curves corresponds to the increase of the wall temperature, provided that it is larger than the heat carrier temperature

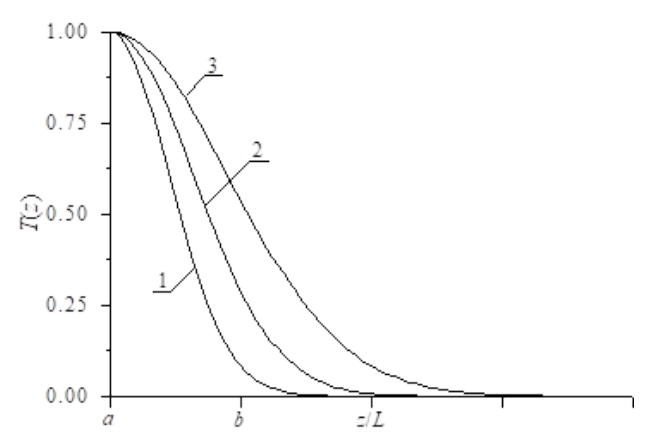

Fig. 4. The dependence of the temperature of the heat carrier on the axial at various values of the temperature of the section of the transport system. The increasing number of curves corresponds to the increase of the temperature of an external section, provided that it is less than the heat carrier temperature 


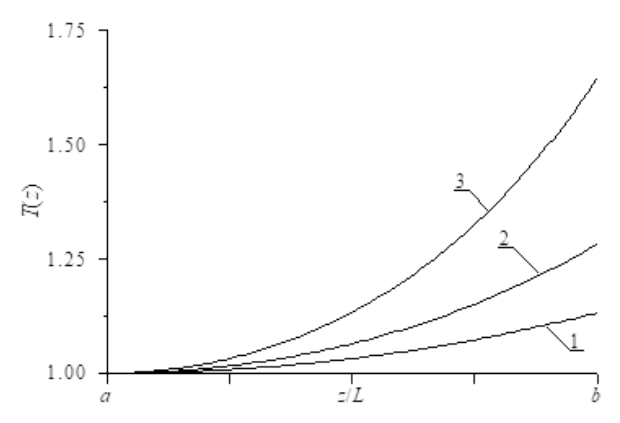

Fig. 5. The dependence of the temperature of the heat carrier on the axial coordinate at various values of the temperature of the section of the transport system. The increasing number of curves corresponds to the increase of the considered section, provided that it is larger than the heat carrier temperature

\section{Conclusion}

In this paper, an approach to changing the temperature of liquids and gases in a pipeline by using metal porous sections with the modification of temperature by external actions was considered. The model for the prediction of mass and heat transport in the investigated pipeline was also introduced. Finally, an analytical approach of analysis mass and heat transport was considered. The analysis of mass and heat transport shows that the developed model is qualitatively adequate.

\section{References}

Carslaw, H.S., \& Jaeger, J.C. (1964). Conduction of heat in solids. Clarendon Press.

Fedorenko, B.Z., Gorlov, A.C., \& Petrashev, V.I. (2020). Optimization of heat exchange in chain curtains of cement furnaces: heat exchange at the evaporation of moisture from sludge with a droping rate. Applied Mathematics and Physics, 52(4), $262-270$.

Kalaev, V. (2020). Computer modeling of HMCz Si growth. Journal of the Crystal Growth, 532, 125413.

Liu, X., Harada, H., Miyamura, Y., Han, X., Nakano, S., Nishizawa, S., \& Kakimoto, K. (2020). Transient global modeling for the pulling process of Czochralski silicon crystal growth. I. Principles, formulation, and implementation of the model. Journal of the Crystal Growth, 532, 125404.

Pankratov, E.L. (2012). Decreasing of Depth of $p$ - $n$-Junction in a semiconductor heterostructure by serial radiation processing and microwave annealing. Journal of Computational and Theoretical Nanoscience, 9(1), 41-49.

Pankratov, E.L., \& Bulaeva, E.A. (2013). Optimal criteria to estimate temporal characteristics of diffusion process in a media with inhomogenous and nonstationary parameters. Analysis of Influence of Variation of Diffusion Coefficient on Values of Time Characteristics. Reviews in Theoretical Science, 1(3), 307-318.

Sokolov, Y.D. (1955). About the definition of dynamic forces in the mine lifting. Applied Mechanics, 1(1), 23-35. 
